\title{
A sodium-glucose co-transporter 2 inhibitor empagliflozin prevents abnormality of circadian rhythm of blood pressure in salt-treated obese rats
}

\begin{abstract}
Yui Takeshige ${ }^{1,2}$, Yoshihide Fujisawa ${ }^{3}$, Asadur Rahman ${ }^{1}$, Wararat Kittikulsuth ${ }^{1}$, Daisuke Nakano ${ }^{1}$, Hirohito Mori ${ }^{4}$, Tsutomu Masaki ${ }^{4}$, Koji Ohmori ${ }^{5}$, Masakazu Kohno ${ }^{5}$, Hiroaki Ogata ${ }^{2}$ and Akira Nishiyama ${ }^{1}$

Studies were performed to examine the effects of the selective sodium-glucose co-transporter 2 (SGLT2) inhibitor empagliflozin on urinary sodium excretion and circadian blood pressure in salt-treated obese Otsuka Long Evans Tokushima Fatty (OLETF) rats. Fifteen-week-old obese OLETF rats were treated with $1 \% \mathrm{NaCl}$ (in drinking water), and vehicle $(0.5 \%$ carboxymethylcellulose, $n=10$ ) or empagliflozin ( $10 \mathrm{mg} \mathrm{kg}^{-1}$ per day, p.o., $n=11$ ) for 5 weeks. Blood pressure was continuously measured by telemetry system. Glucose metabolism and urinary sodium excretion were evaluated by oral glucose tolerance test and high salt challenge test, respectively. Vehicle-treated OLETF rats developed non-dipper type blood pressure elevation with glucose intolerance and insulin resistance. Compared with vehicle-treated animals, empagliflozin-treated OLETF rats showed an approximately 1000 -fold increase in urinary glucose excretion and improved glucose metabolism and insulin resistance. Furthermore, empagliflozin prevented the development of blood pressure elevation with normalization of its circadian rhythm to a dipper profile, which was associated with increased urinary sodium excretion. These data suggest that empagliflozin elicits beneficial effects on both glucose homeostasis and hypertension in salt-replete obese states.
\end{abstract}

Hypertension Research (2016) 39, 415-422; doi:10.1038/hr.2016.2; published online 28 January 2016

Keywords: circadian blood pressure; empagliflozin; obese; sodium-glucose co-transporter 2 (SGLT2); urinary sodium excretion

\section{INTRODUCTION}

Hypertension and obesity frequently occur together, exposing patients to increased metabolic risks, particularly patients with metabolic syndrome and type 2 diabetes mellitus. ${ }^{1,2}$ Epidemiological studies have shown that more than $75 \%$ of hypertensive patients are overweight or obese. ${ }^{3-5}$ Furthermore, obesity may render hypertensive patients treatment resistant in metabolic syndrome and type 2 diabetes mellitus. ${ }^{1}$

Recently, sodium-glucose co-transporter 2 (SGLT2) inhibitors were approved for use as antihyperglycemic drugs. ${ }^{6}$ SGLT2 inhibitors lower blood glucose levels by reducing renal reabsorption of glucose at the S1 segment of the proximal tubules. ${ }^{7}$ In addition to having a low risk of hypoglycemia, ${ }^{8}$ accumulating clinical evidence has revealed that treatment with SGLT2 inhibitors elicits a significant blood pressure reduction. ${ }^{6,9,10}$ A meta-analysis of 21 placebo-controlled studies published in April 2013 showed a significant blood pressure reduction by treatment with SGLT2 inhibitors in patients with type 2 diabetes. ${ }^{11}$ Another meta-analysis and a pre-specified pool analysis of 12 placebocontrolled studies have also shown similar blood pressure lowering effects of SGLT2 inhibitors. ${ }^{6}$ To directly demonstrate the blood pressure lowering effect of SGLT2 inhibition, clinical trials with pre-specified primary end points of changes in systolic blood pressure (NCT01195662, NCT01939496) have just been completed and data will be presented in the near future.

Although the precise mechanism responsible for SGLT2 inhibitorinduced blood pressure reduction is not clear, possible hypotheses include the improvement of glucose metabolism and insulin resistance, as well as concomitant weight loss of patients with the SGLT2 inhibitors. ${ }^{6,8,10}$ However, clinical data have shown that during treatment with SGLT2 inhibitors, reductions in blood pressure were not correlated with changes in HbAlc levels and body weight, suggesting that SGLT2 inhibitors have blood pressure lowering effects beyond glycemic control and weight loss. ${ }^{6,12}$ Therefore, Lambers-Heerspink et al. ${ }^{13}$ examined the effects of an SGLT2 inhibitor, dapagliflozin and hydrochlorothiazide in patients with type 2 diabetes. They showed that both dapagliflozin- and hydrochlorothiazide-induced blood pressure reductions were associated with reductions in plasma volume, suggesting that dapagliflozin may have a diuretic-like capacity to lower the blood pressure. Nevertheless, there is no clinical report of hyponatremia with the use of SGLT2 inhibitors. ${ }^{6}$ In addition,

\footnotetext{
${ }^{1}$ Department of Pharmacology, Faculty of Medicine, Kagawa University, Kagawa, Japan; ${ }^{2}$ Division of Nephrology, Department of Medicine, Showa University School of Medicine, Yokohama, Japan; ${ }^{3}$ Life Science Research Center, Faculty of Medicine, Kagawa University, Kagawa, Japan; ${ }^{4}$ Department of Gastroenterology, Faculty of Medicine, Kagawa University, Kagawa, Japan and ${ }^{5}$ Department of Cardiology, Faculty of Medicine, Kagawa University, Kagawa, Japan

Correspondence: Dr A Nishiyama, Department of Pharmacology, Faculty of Medicine, Kagawa University, 1750-1 Ikenobe, Miki-cho, Kita-gun, Kagawa 761-0793, Japan.

E-mail: akira@kms.ac.jp

Received 31 July 2015; revised 25 November 2015; accepted 28 November 2015; published online 28 January 2016
} 
preclinical studies failed to show a significant increase in urinary sodium excretion after chronic treatment with SGLT2 inhibitors, ${ }^{14,15}$ probably owing to the technical difficulties in the precise evaluation of urinary sodium excretion under experimental conditions without any dietary intervention. ${ }^{16}$

Here, we aimed to investigate whether an SGLT2 inhibitor actually elicits blood pressure reduction with natriuresis. For this purpose, we used a telemetry technique ${ }^{17}$ and sodium challenge test ${ }^{18}$ for measuring 24-h blood pressure and urinary sodium excretion, respectively. Recent studies have shown that in obese subjects, elevated sodium sensitivity of blood pressure contributes to abnormality of blood pressure circadian rhythm. ${ }^{19,20}$ Therefore, we examined the effect of the selective SGLT2 inhibitor, empagliflozin, ${ }^{21}$ on urinary sodium excretion and circadian blood pressure in salt-treated obese Otsuka Long Evans Tokushima Fatty (OLETF) rats. ${ }^{22}$

\section{METHODS}

\section{Experimental animals}

Rats were maintained in a temperature-controlled $\left(24 \pm 2{ }^{\circ} \mathrm{C}\right)$ room with a 12 -h light/dark cycle and $55 \pm 5 \%$ humidity. Experimental protocols and animal care were performed according to the guidelines for the care and use of animals established by Kagawa University, Japan.

Experiments were performed in 21 male 10-week-old OLETF rats and 8 age-matched lean Long-Evans Tokushima Otsuka (LETO) rats (Hoshino Laboratory Animals, Sakai-Higashi, Japan). ${ }^{22}$ At 13 weeks of age, the radiotelemetry device was implanted under isoflurane anesthesia. After 2 weeks of acclimatization, all animals were treated with $1 \% \mathrm{NaCl}$ in drinking water and normal salt diet $(0.5 \% \mathrm{NaCl}$, SLC Japan, Hamamatsu, Japan) from 15 weeks of age. LETO rats were also treated with vehicle $(0.5 \%$ methyl cellulose; Nacalai Tesque, Kyoto, Japan), while OLETF rats were randomly divided into two groups for treatment with vehicle $(n=10)$ or the selective SGLT2 inhibitor, empagliflozin $\left(10 \mathrm{mg} \mathrm{kg}^{-1}\right.$ per day; p.o., $\left.n=11\right)$. The dose of empagliflozin was determined on the basis of previous studies in rats. ${ }^{23,24}$ Animals underwent treatment for 5 weeks. Mean arterial pressure (MAP) was measured in conscious rats by the telemetry device. ${ }^{17}$ Twenty-four-hour urine samples were also collected every $2-3$ weeks using metabolic cages.

\section{Oral glucose tolerance test}

At 20 weeks of age, an oral glucose tolerance test (OGTT) was performed with a $2 \mathrm{~g} \mathrm{~kg}^{-1}$ body weight glucose feeding by gavage, as previously described. ${ }^{22}$ Animals were food restricted overnight before the OGTT. Blood was drawn from a cut at the tip of the tail before and at 15, 30, 60 and 90 min after the glucose feeding, and blood glucose levels were analyzed by glucometer. Whole blood was mixed thoroughly with ethylene-diaminetetraacetic acid and centrifuged to separate the plasma. Plasma samples were analyzed to determine the insulin concentration.

\section{Sodium challenge test}

After the OGTT test, $1 \% \mathrm{NaCl}$ drinking water was switched to tap water. Furthermore, all animals were treated with low sodium diet $(0.03 \% \mathrm{NaCl}$, SLC Japan) for 1 week, while treatment with vehicle or empagliflozin was continued. Then, a sodium challenge test was performed with a $1 \mathrm{~g} \mathrm{~kg}^{-1}$ body weight $\mathrm{NaCl}$ feeding by gavage, as previously described..$^{18}$ Urine samples were collected every $12 \mathrm{~h}$ using metabolic cages, for up to $60 \mathrm{~h}$.

\section{Sample preparation}

After finishing the sodium challenge test, animals were maintained with normal salt diet $(0.5 \% \mathrm{NaCl})$ and tap water for 3 days until 22 weeks of age. Thereafter, animals were fasted overnight and blood was collected from the abdominal aorta under sodium pentobarbital anesthesia $\left(65 \mathrm{mg} \mathrm{kg}^{-1}\right)$ at $0900-1200 \mathrm{~h}$. Then, the right renal artery was clamped, and renal cortical tissue from the right kidney was collected in RNAlater (Life Technologies, Carlsbad, CA, USA) and stored overnight at $4{ }^{\circ} \mathrm{C}$, then subsequently snap frozen in liquid nitrogen and stored at $-80^{\circ} \mathrm{C}$ until processing for RNA extraction. Left kidneys were perfused with saline and harvested for histological analyses, as previously described. ${ }^{22}$

\section{Telemetry system}

To measure MAP in conscious animals, we used a telemetry technique, as described previously. ${ }^{17}$ Under isoflurane anesthesia, an abdominal incision was made for implantation of the radio-telemetry device. The system consists of a radiofrequency transmitter (TA11PA-C40) with a receiver panel (RPC-1) and an adaptor (R11CPA) with an ambient pressure monitor (APR-1) (Data Science International, St Paul, MN, USA). Data were collected and analyzed using Dataquest ART version 4.3 (Data Science International). Two weeks after surgery, baseline MAP was measured. All animals underwent a 24-h acclimatization period on telemetry receiver panel, and vehicle and empagliflozin were administered $3 \mathrm{~h}$ before the start 24 -h blood pressure measurement. To evaluate the circadian rhythm of blood pressure, we calculated the value of 12-h dark period $(1800-0500 \mathrm{~h})$ and 12-h light period $(0600-1500 \mathrm{~h}){ }^{17}$

\section{Real-time reverse-transcription PCR}

RNA was isolated from renal cortical tissue by the phenol-chloroform extraction method and cDNA prepared, as previously described. ${ }^{25}$ The mRNA expression of SGLT1 and SGLT2 was analyzed by real-time PCR using an ABI Prism 7000 with Power SYBR Green PCR Master Mix (Applied Biosystems, Foster City, CA, USA). The following primers were used: sense $5^{\prime}$-TCT TCGCTATCAGCGTCGTC- $3^{\prime}$ and anti-sense $5^{\prime}$-TGCGCTCTTCTGTGCTG TTA-3' for rat SGLT1; sense 5'-GACATTCTGGTCATTGCCGC-3' and anti-sense $5^{\prime}$-CTGCCAAGAAGTAGCCACCA- $3^{\prime}$ for rat SGLT2; sense $5^{\prime}$-GAA CTTGGCATTGAGGAGTCT-3' and anti-sense $5^{\prime}$-GTGATCGAACCTTTC CAGTGC-3' for rat CLOCK; sense 5'-TCCGATGACGAACTGAAACAC-3' and anti-sense $5^{\prime}$-CTCGGTCACATCCTACGACAA- $3^{\prime}$ for rat bmall; sense 5'-CCAGGCCCGGAGAACCTTTTT-3' and anti-sense 5'-CGAAGTTTGAGC TCCCGAAGT-3' for rat per1; sense 5'-GCAGCCTTTCGATTATTCTTC-3' and anti-sense $5^{\prime}$-GCTCCACGGGTTGATGAAG- $3^{\prime}$ for rat per2. The oligonucleotide primer sequences for $\beta$-actin are described in previous studies. ${ }^{25}$ All data are presented as the relative differences between the vehicle-treated LETO rats and the groups of OLETF rats after normalization to $\beta$-actin gene expression.

\section{Histological examination}

The left kidney was fixed with $10 \%$ formalin ( $\mathrm{pH}$ 7.4), embedded in paraffin, sectioned into $3-\mu \mathrm{m}$-thick slices and stained with PAS (periodic acid-Schiff). The extent of glomerular sclerotic changes was semi-quantitatively evaluated by an automatic image analysis system using PAS-stained sections, as previously described. ${ }^{25}$ Immunostaining of SGLT2 was also performed in formalin-fixed paraffin-embedded kidney tissues. After paraffin sections were deparaffinized and autoclaved in $10 \mathrm{mmol}^{-1}$ sodium citrate buffer ( $\mathrm{pH}$ 6.0) for antigen retrieval, $10 \%$ normal goat serum (Nichirei Biosciences, Tokyo, Japan) was added to the sections to block non-specific binding. The sections were then incubated with an anti-SGLT2 antibody (dilution 1:200, ab85626, Abcam, Cambridge, UK). Sections were subsequently incubated with the secondary antibody (Histofine Simple Stain Rat MAX-PO, Nichirei Biosciences).

\section{Other analytical procedures}

Urine osmolality was determined by freezing point depression (Osmett II; Precision System, Natick, MA, USA). Urinary albumin (Shibayagi, Shibukawa, Japan), plasma insulin (Morinaga Institute of Biological Science, Yokohama, Japan), plasma aldosterone (Cayman Chemical, Ann Arbor, MI, USA) and urinary angiotensinogen (Takara Bio, Kusatsu, Japan) were measured using commercially available assay kits. Plasma total cholesterol, triglycerides, glycoalbumin and creatinine were measured by an automated analyzer (7020-Automatic Analyzer, Hitachi High-Technologies, Tokyo, Japan).

\section{Statistical analysis}

Data are presented as the means \pm s.e. Statistical comparisons of the differences between values were performed using one- or two-way analysis of variance 
Table 1 Body weight and metabolic parameters

\begin{tabular}{|c|c|c|c|c|}
\hline & Sampling period (weeks of age) & LETO rats+vehicle & OLETF rats+vehicle & OLETF rats+empagliflozin \\
\hline Food intake (g per day) & 20 & $20 \pm 2^{*}$ & $23 \pm 2^{*}$ & $26 \pm 2^{*, \dagger}$ \\
\hline Body weight (g) & 20 & $446 \pm 6$ & $572 \pm 21^{*}$ & $539 \pm 18^{*}$ \\
\hline \multirow[t]{2}{*}{ PPBG (mg dl-1) } & 15 & $94 \pm 5$ & $104 \pm 6$ & $112 \pm 5$ \\
\hline & 20 & $97 \pm 3$ & $108 \pm 6$ & $109 \pm 5$ \\
\hline Triglycerides $\left(\mathrm{mg} \mathrm{dl}^{-1}\right)$ & 22 & $55 \pm 12$ & $124 \pm 36^{*}$ & $122 \pm 20^{*}$ \\
\hline Creatinine $\left(\mathrm{mg} \mathrm{dl}^{-1}\right)$ & 22 & $0.30 \pm 0.04$ & $0.32 \pm 0.01$ & $0.30 \pm 0.01$ \\
\hline Urinary albumin excretion (mg per day) & 20 & $0.7 \pm 0.2$ & $140 \pm 25^{*}$ & $108 \pm 13^{*}$ \\
\hline
\end{tabular}

Abbreviations: Cr, creatinine; LETO rats, Long Evans Tokushima Otsuka rats; OLETF rats, Otsuka Long Evans Tokushima Fatty rats; PPBG, postprandial blood glucose.

${ }^{\star} P<0.05$ vs. LETO rats+vehicle.

$\dagger P<0.05$; OLETF rats+vehicle vs. OLETF rats+empagliflozin.

combined with the Newman-Keuls post hoc test. $P$-values of $<0.05$ were considered as statistically significant.

\section{RESULTS}

Metabolic parameters and urinary glucose excretion

Food intake and body weight were greater in obese OLETF rats than in lean LETO rats throughout the observation period. Treatment with empagliflozin increased food intake but did not change the body weight in salt-treated OLETF rats (Table 1). Postprandial blood glucose (PPBG) and plasma glycoalbumin levels tended to be higher in OLETF than in LETO rats; however, these changes were not statistically significant. Treatment with empagliflozin did not change PPBG and plasma glycoalbumin levels in OLETF rats (Table 1). Both plasma total cholesterol and triglycerides levels were significantly higher in OLETF than in LETO rats, but empagliflozin did not significantly change these values. Salt-treated OLETF rats showed obvious albuminuria at 20 weeks of age. Treatment with empagliflozin tended to decrease urinary albumin excretion, but these changes were not statistically significant. In contrast, plasma creatinine levels were not different among the groups (Table 1). Similarly, the glomerular PAS-positive area was similar among the groups (data not shown), indicating that renal tissue injury is not obvious in salt-treated obese OLETF rats at this age.

Figure 1 shows the temporal profiles of urinary glucose excretion. At baseline (15 weeks of age), both LETO and OLETF rats showed barely any glycosuria. During treatment with $1 \% \mathrm{NaCl}$, the urinary excretion rate of glucose was not changed in LETO and OLETF rats $(1.7 \pm 0.5$ and $1.3 \pm 0.2 \mathrm{mg}$ per day at 20 weeks of age, respectively). However, the urinary excretion rate of glucose was approximately 1000 -fold increased by administration of empagliflozin in salt-treated OLETF rats $(1297.9 \pm 107.5 \mathrm{mg}$ per day at 20 weeks of age).

OGTT studies demonstrated that salt-treated OLETF rats showed a significantly greater area under the curve (AUC) of blood glucose and plasma insulin as compared with salt-treated LETO rats. Furthermore, empagliflozin caused a significant decrease in the AUC of blood glucose and plasma insulin in salt-treated OLETF rats (Figures 2a-d).

\section{Mean arterial blood pressure}

Temporal profiles of averaged 24-h MAP are shown in Figure 3. At baseline, obese OLETF rats showed significantly higher MAP than lean LETO rats $(107 \pm 1$ vs. $100 \pm 3 \mathrm{~mm} \mathrm{Hg}$ at 15 weeks of age, respectively). Treatment with salt elevated the MAP in OLETF rats, but not in LETO rats $(113 \pm 1$ vs. $102 \pm 2 \mathrm{~mm} \mathrm{Hg}$ at 20 weeks of age, respectively). In obese OLETF rats, administration of empagliflozin prevented the development of salt-induced blood pressure elevation.

\section{Circadian rhythm of arterial blood pressure}

The circadian rhythm of MAP is shown in Figure 4. At 15 weeks of age, the baseline dark period MAP was significantly higher as compared with light period MAP in both LETO (106 \pm 2 vs. $101 \pm 2 \mathrm{~mm} \mathrm{Hg}, \quad P<0.05)$ and OLETF rats $(110 \pm 1$ vs. $106 \pm 1 \mathrm{~mm} \mathrm{Hg}, P<0.05)$, indicating the dipper-type phenomenon of blood pressure. ${ }^{17,26,27}$ Administration of $1 \% \mathrm{NaCl}$ for 5 weeks did not change the dipping pattern of MAP in LETO rats $(108 \pm 3 v$ s. $102 \pm 2 \mathrm{~mm} \mathrm{Hg}$, during dark and light period MAP at 20 weeks of age, respectively, Figure 4a). Similarly, the dipping pattern of MAP was not changed by salt treatment for 3 weeks in OLETF rats. However, after 4 weeks of salt administration, the differences in the MAP between dark and light period disappeared in OLETF rats $(113 \pm 1 v s$. $112 \pm 2 \mathrm{~mm} \mathrm{Hg}$ during dark and light period MAP at 20 weeks of age, respectively, Figure $4 \mathrm{~b}$ ), indicating a non-dipper type of blood pressure elevation. ${ }^{26,27}$ Notably, administration of empagliflozin normalized dipping pattern of blood pressure in salt-treated obese OLETF rats. Specifically, throughout the observation period in empagliflozin-treated animals, MAP was significantly higher during the dark period compared with the light period $(110 \pm 2 v s$. $104 \pm 2 \mathrm{~mm} \mathrm{Hg}$ at 20 weeks of age, respectively, Figure 4c). Changes in delta difference between dark and light period MAP are depicted in Figures $4 \mathrm{~d}$ and $\mathrm{e}$.

\section{Urinary osmolality and sodium challenge test}

As shown in Figure 5a, urinary osmolality was higher in LETO than in OLETF rats throughout the observation period. Administration of empagliflozin did not significantly change urinary osmolality in salt-treated OLETF rats. Data obtained from the sodium challenge test are shown in Figure 5b. After acute administration of $\mathrm{NaCl}$ ( $1 \mathrm{~g} \mathrm{~kg}^{-1}$, p.o.), the 0 - to 12 -h urinary sodium excretion rate was similar between OLETF and LETO rats. However, treatment with empagliflozin significantly increased the 0 - to 12 -h urinary sodium excretion in OLETF rats $(P<0.05)$. Similarly, the 12 - to 24 -h urinary sodium excretion was also increased by empagliflozin $(P<0.05)$. 


\section{SGLT2, SGLT1 and clock genes in the kidney}

After finishing the sodium challenge test, animals were maintained on a normal salt diet and tap water for 3 days then renal cortical tissues were harvested for measuring mRNA levels at 22 weeks of age. There were no significant differences in mRNA levels of SGLT2 and SGLT1 among the groups (Figures $6 \mathrm{a}$ and $\mathrm{b}$ ). Immunohistochemistry revealed that SGLT2 staining in the brush borders of proximal tubules was stronger in OLETF rats than in LETO rats. In contrast, SGLT2 immunostaining was faint in empagliflozin-treated rats (Figure 6c). On the other hand, gene expression of clock genes, including clock, bmal1, per1 and per2, in renal cortical tissues was not different among the groups (data not shown).

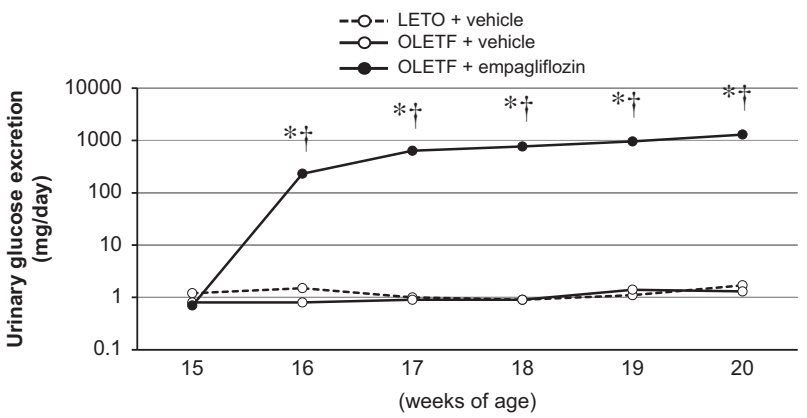

Figure 1 Temporal profiles of urinary glucose excretion. Urinary glucose excretion was increased approximately 1000 -fold by administration of empagliflozin in salt-treated Otsuka Long Evans Tokushima Fatty (OLETF) rats. LETO rats, Long Evans Tokushima Otsuka rats. ${ }^{*} P<0.05$ vs. LETO rats+vehicle. ${ }^{\dagger} P<0.05$; OLETF rats+vehicle vs. OLETF rats+empagliflozin. AUC, area under the curve.

\section{Urinary angiotensinogen and plasma aldosterone}

At 20 weeks of age, urinary angiotensinogen excretion was significantly higher in salt-treated OLETF rats than in salt-treated LETO rats $(177 \pm 60$ vs. $442 \pm 39 \mathrm{ng}$ per day, $P<0.05)$. Treatment with empagliflozin slightly but significantly increased urinary angiotensinogen excretion in salt-treated OLETF rats $(678 \pm 65 \mathrm{ng}$ per day, $P<0.05)$. At 22 weeks of age (animals were maintained on a normal salt diet and tap water for 3 days after finishing the sodium challenge test), plasma aldosterone levels were not different between LETO and OLETF rats $\left(239 \pm 42\right.$ vs. $\left.283 \pm 51 \mathrm{pg} \mathrm{ml}^{-1}\right)$. Treatment with empagliflozin did not change plasma aldosterone level in OLETF rats $\left(308 \pm 59 \mathrm{pg} \mathrm{ml}^{-1}\right)$.

\section{DISCUSSION}

The present study has demonstrated that SGLT2 inhibitor-induced blood pressure reduction was accompanied by normalization of blood pressure dipping pattern in salt-treated obese rats. Our data also revealed that these effects of an SGLT2 inhibitor were associated with an increase in urinary sodium excretion. To our knowledge, these data indicate for the first time that in addition to the control of glucose metabolism, treatment with an SGLT2 inhibitor elicits beneficial effects on circadian rhythm of blood pressure in salt-replete obese states.

In the present study, young obese OLETF rats showed similar MAP to lean LETO rats before high salt treatment. In these animals, the MAP was significantly higher in the dark time compared with the light time. These data are similar to those in previous reports showing that MAP was higher at dark time than light time in normotensive rats. ${ }^{17,26,27}$ In the present study, treatment with salt for 3 weeks did not change day time MAP, but significantly increased night time MAP in obese OLETF rats, whereas MAP was not changed by salt treatment in lean LETO rats. These data are similar to those observed in previous
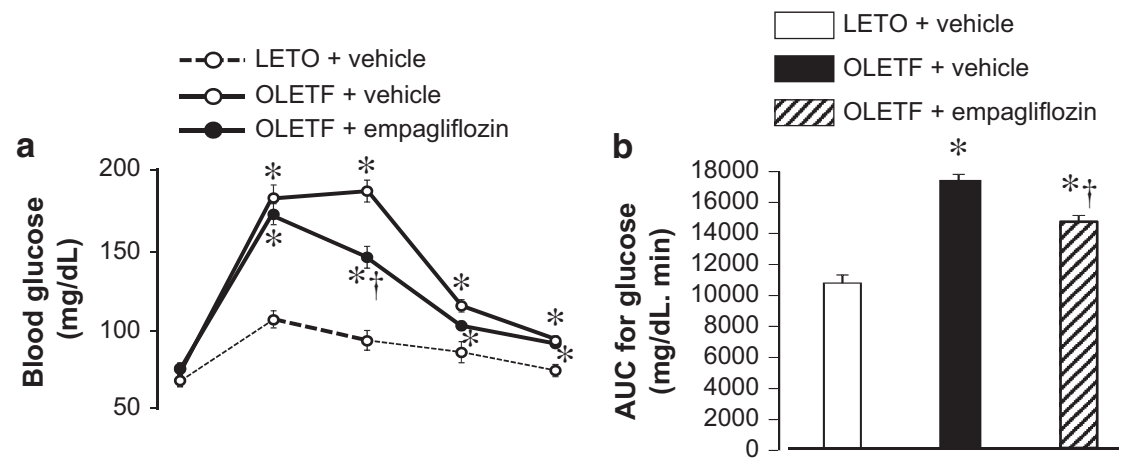

C

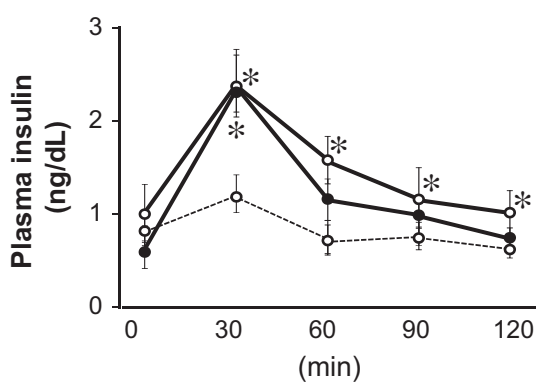

d

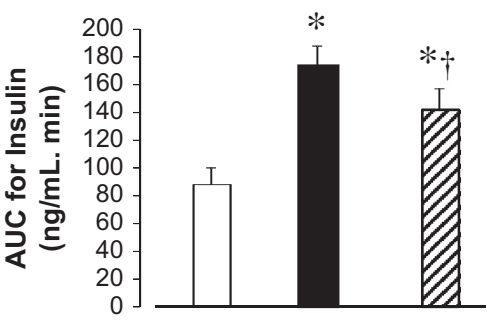

Figure 2 Blood glucose and insulin levels and their respective area under the curve (AUC) during the oral glucose tolerance test (OGTT). (a, b) Blood glucose and the AUC at 20 weeks of age. At baseline, blood glucose levels were not different among the groups (a). Otsuka Long Evans Tokushima Fatty (OLETF) rats demonstrated a greater AUC, which was suppressed by empagliflozin (b). (c, d) Plasma insulin levels and the AUC at 20 weeks of age. Baseline plasma insulin levels were not different among the groups (c). OLETF rats demonstrated a greater AUC (d), which was suppressed by treatment with empagliflozin. ${ }^{*} P<0.05$ vs. LETO rats+vehicle. ${ }^{\dagger} P<0.05$; OLETF+vehicle vs. OLETF+empagliflozin. LETO, Long Evans Tokushima Otsuka. 


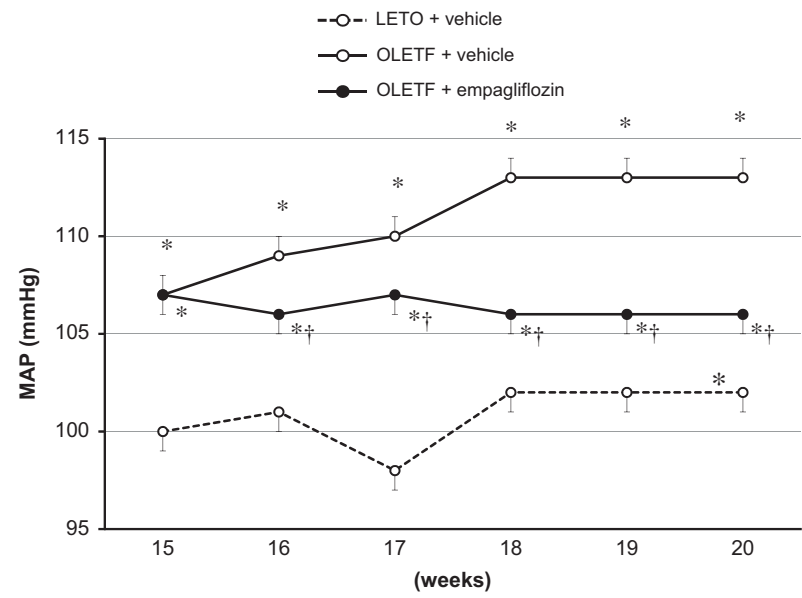

Figure 3 Temporal profiles of averaged 24-h MAP measured by telemetry system. At baseline, obese Otsuka Long Evans Tokushima Fatty (OLETF) rats showed significantly higher mean arterial pressure (MAP) than lean Long Evans Tokushima Otsuka (LETO) rats. Treatment with salt elevated the MAP in OLETF rats, but not in LETO rats. Treatment with empagliflozin prevented the development of hypertension in salt-treated OLETF rats. ${ }^{*} P<0.05$ vs. LETO rats+vehicle. ${ }^{\dagger} P<0.05$; OLETF+vehicle vs. OLETF+empagliflozin. studies where acute salt feeding for 7 days significantly increased night time MAP with an extreme dipping pattern of blood pressure in Dahl salt-sensitive hypertensive rats. ${ }^{17}$ Interestingly, we found that further continuous treatment with a high salt diet also increased daytime MAP in obese OLETF rats, leading to non-dipper type of blood pressure elevation. These data support the concept based on the clinical studies ${ }^{1,2,19,20}$ that salt-dependent hypertension is associated with disrupted circadian rhythm of blood pressure in obese subjects. On the other hand, renal cortical expression of clock gene was not different between the animals. Thus, the present study failed to show any evidence regarding the possible contribution of clock gene in the kidney to the abnormality of circadian rhythm of blood pressure.

In the present study, the selective SGLT2 inhibitor empagliflozin ${ }^{21}$ prevented the development of blood pressure elevation and disruption of circadian rhythm of blood pressure. To test the hypothesis that empagliflozin-induced blood pressure reduction is associated with natriuresis, we tried to estimate a cumulative sodium balance. Unfortunately, our metabolic cage system could not avoid the loss of water, because OLETF rats severely spilled over a drinking water containing $1.0 \% \mathrm{NaCl}$. On the other hand, further investigation with a sodium challenge test ${ }^{18}$ revealed that treatment with an SGLT2 inhibitor actually elicited an increase in urinary excretion rate of
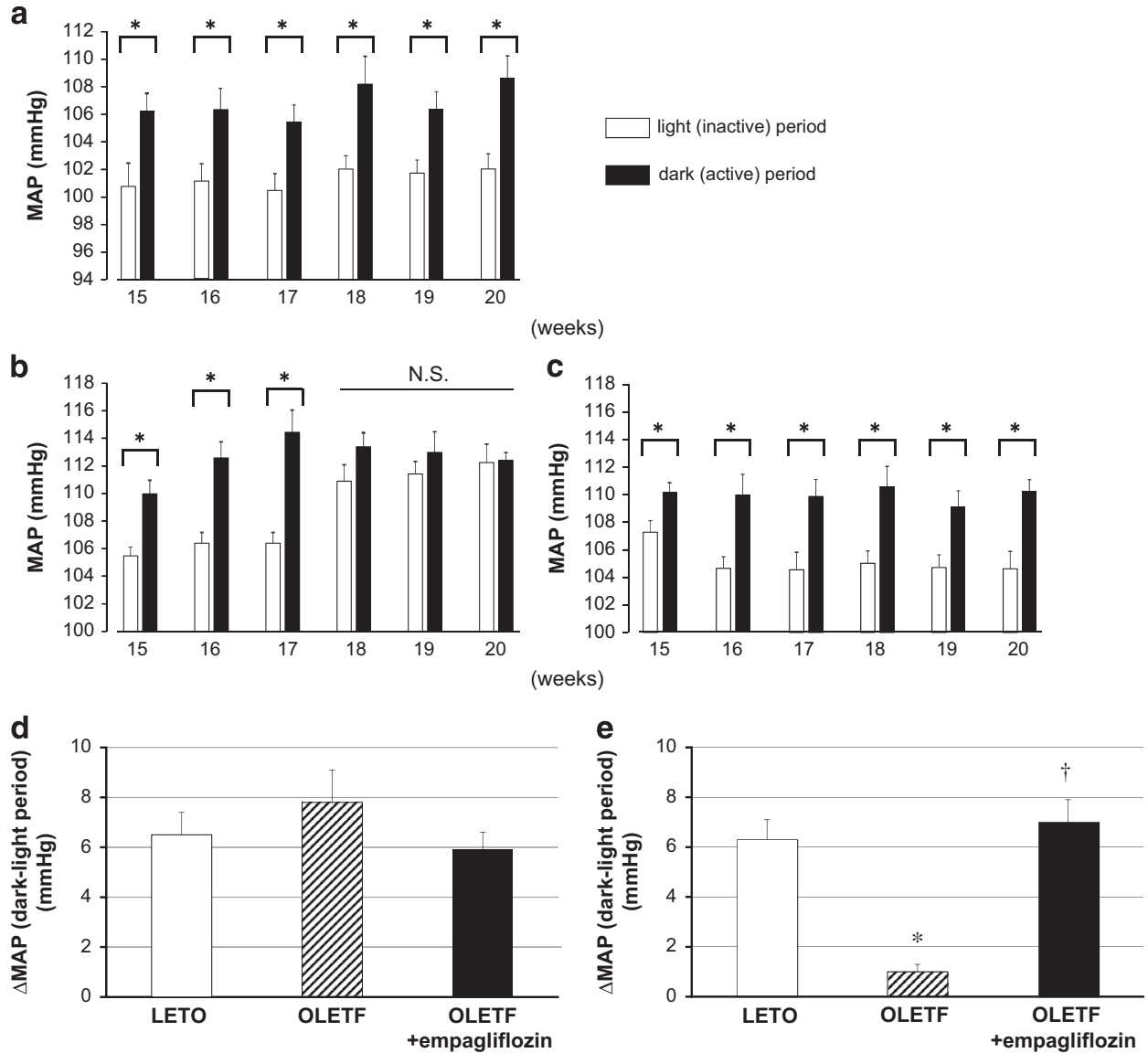

Figure 4 Circadian rhythms of mean arterial pressure (MAP) measured hourly by a telemetry system in vehicle-treated Long Evans Tokushima Otsuka (LETO) (a), vehicle-treated Otsuka Long Evans Tokushima Fatty (OLETF) (b) and empagliflozin-treated OLETF rats (c). Baseline MAP was higher during the dark period compared with the light period in both LETO and OLETF rats. Four weeks after salt treatment, the differences in the MAP between dark and light period disappeared in OLETF rats. Empagliflozin prevented abnormality of blood pressure circadian rhythm in salt-treated OLETF rats. Changes in delta difference between dark and light period MAP at 15 (d) and 20 (e) weeks of age. ${ }^{*} P<0.05$ vs. LETO rats+vehicle. ${ }^{\dagger} P<0.05$; OLETF rats+vehicle $v s .0 L E T F$ rats+empagliflozin. 
sodium in obese OLETF rats. These data are in agreement with those observed in sodium-sensitive hypertensive patients, such as those with obesity and metabolic syndrome, where sodium restriction shifted the circadian rhythm of blood pressure from non-dipper to dipper. ${ }^{28,29}$ Since high prevalence rates of cardiovascular complications including stroke and myocardial injury are associated with a non-dipper pattern of blood pressure in hypertensive patients, ${ }^{1,2}$ blood pressure control

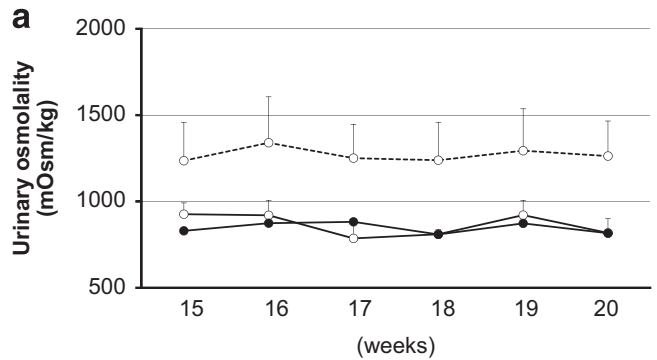

$$
\text { b }
$$

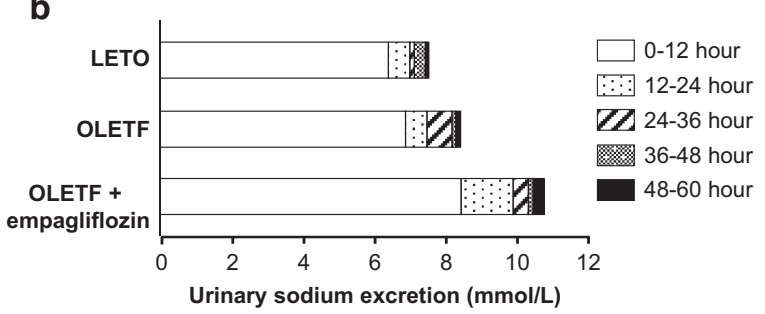

Figure 5 (a) Temporal profiles of urinary osmolality. Urinary osmolality was higher in Long Evans Tokushima Otsuka (LETO) than in Otsuka Long Evans Tokushima Fatty (OLETF) rats throughout the observation period. Administration of empagliflozin did not significantly change urinary osmolality in salt-treated OLETF rats. (b) Urinary sodium excretion evaluated by a sodium challenge test. After acute administration of $\mathrm{NaCl}\left(1 \mathrm{~g} \mathrm{~kg}^{-1}\right.$, p.o.), 0- to 12- and 12- to 24-h urinary sodium excretion rates were similar between OLETF and LETO rats. However, treatment with empagliflozin significantly increased 0 - to 12 - and 12 - to $24-\mathrm{h}$ urinary sodium excretion rates in OLETF rats $(P<0.05)$.
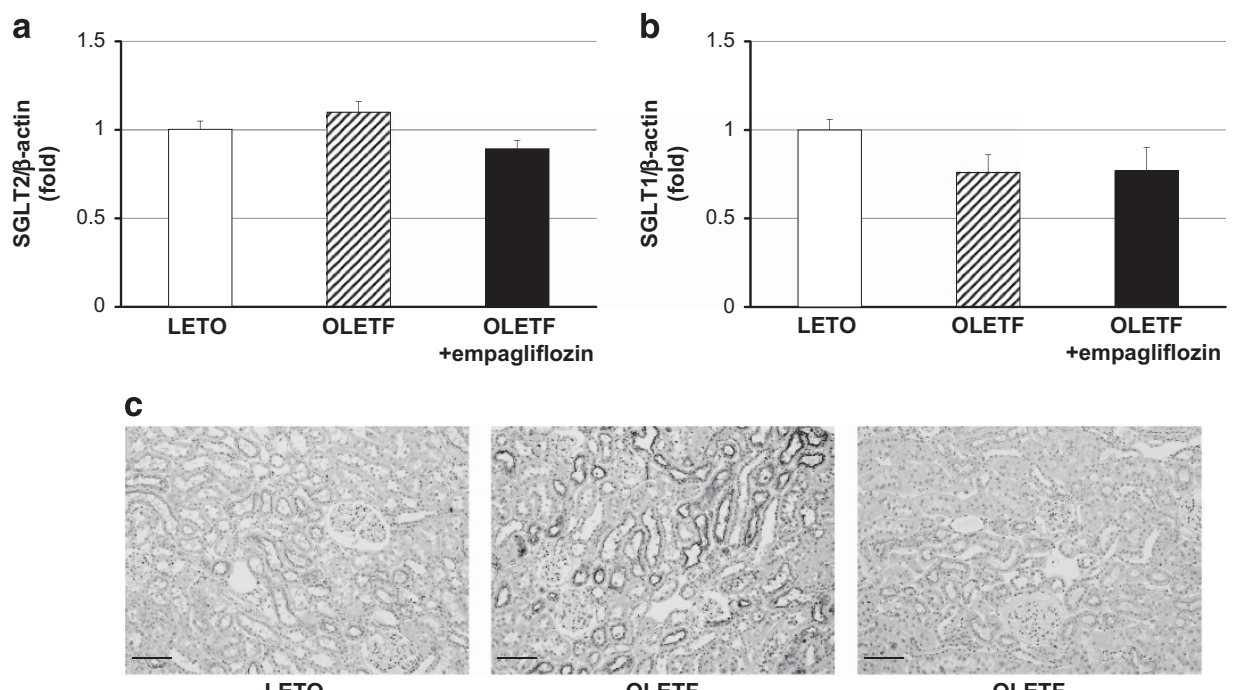

LETO
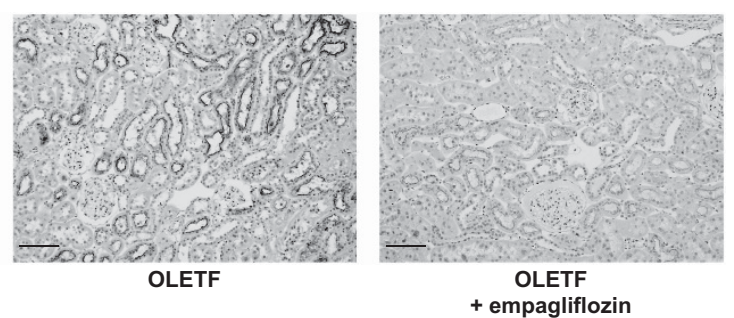

Figure 6 Gene expression of sodium-dependent glucose co-transporter 2 (SGLT2, a) and sodium-dependent glucose co-transporter 1 (SGLT1, b) in renal cortical tissues. There were no significant differences in renal cortical mRNA levels of SGLT2 and SGLT1 among the groups. (c) Representative photomicrograph of SGLT2 immunostaining in the kidney. Bar indicates $100 \mu \mathrm{m}$. SGLT2 staining was obvious in the brush border of proximal tubules in vehicle-treated Otsuka Long Evans Tokushima Fatty (OLETF) rats. Treatment with empagliflozin decreased the immunostaining of SGLT2 in OLETF rats. LETO, Long Evans Tokushima Otsuka. A full color version of this figure is available at the Hypertension Research journal online. 
that treatment with an SGLT2 inhibitor decreases SGLT2 expression in the kidney. However, mRNA levels of SGLT2 in renal cortical tissues were not changed between lean LETO and obese OLETF rats. Furthermore, treatment with an SGLT2 inhibitor did not significantly change these levels in OLETF rats. Although we have no good explanation for the discrepant data between protein and mRNA levels of SGLT2, it may be due to the tissue sampling methods for SGLT2 mRNA levels. SGLT2 is predominantly expressed in the S1 segment of proximal tubules, ${ }^{7}$ but we have harvested renal cortical tissues for SGLT2 mRNA levels. Thus, the relative S1 segment content may be different between each segment of renal cortical tissue, which might affect the values of SGLT2 mRNA levels. Further studies will be needed to determine the expression of SGLT2 and SGLT1 in dissected proximal tubular segments.

Renin-angiotensin-aldosterone system has an important role in the development of hypertension..$^{34,35}$ In the present study, we have measured urinary angiotensinogen as a maker of the activity of renin-angiotensin system in the kidney. ${ }^{34}$ We found that salt-treated obese OLETF rats showed markedly increased urinary angiotensinogen excretion as compared with salt-treated lean LETO rats. In salt-treated OLETF rats, treatment with empagliflozin slightly but significantly increased urinary angiotensinogen excretion, suggesting a possible activation of intrarenal renin-angiotensin system by an SGLT2 inhibitor. These data are consistent with recent clinical data that treatment with empagliflozin significantly increased urinary angiotensinogen excretion in patients with type 1 diabetes. ${ }^{36}$ On the other hand, SGLT2 inhibitor did not change plasma aldosterone level in salttreated OLETF rats. The circadian rhythm of aldosterone is well known, ${ }^{34}$ so further studies are needed to investigate time-dependent changes in plasma levels of aldosterone.

In conclusion, the present study showed that treatment with the SGLT2 inhibitor empagliflozin prevents the development of saltinduced blood pressure elevation and abnormality of blood pressure circadian rhythm, which are associated with an increase in urinary sodium excretion in obese rats. As high prevalence rates of cardiovascular events are associated with disrupted blood pressure circadian rhythm, these data support the postulation that the beneficial effects of SGLT2 inhibitors on glucose metabolism and hypertension provide a further advantage for reducing cardiovascular events.

\section{CONFLICT OF INTEREST}

AN received honoraria for lectures, article contributions or such activities to support promotional activities by Daiichi-Sankyo, Mochida Pharmaceutical and Taisho-Toyama Pharmaceutical. This is a collaborative study with Boehringer Ingelheim (to AN). Other authors declared no conflict of interest.

\section{ACKNOWLEDGEMENTS}

This study was supported in part by the Japan Society for the Promotion of Science (JSPS) Grants-in-Aid for Scientific Research (KAKENHI) (26460343 to AN). Empagliflozin was provided by Boehringer Ingelheim (Ingelheim, Germany). This is a collaborative study with Boehringer Ingelheim (Ingelheim, Germany).

1 Engeli S, Jordan J. Novel metabolic drugs and blood pressure: implications for the treatment of obese hypertensive patients? Curr Hypertens Rep 2013; 15: 470-474.

2 Kario K. Proposal of a new strategy for ambulatory blood pressure profile-based management of resistant hypertension in the era of renal denervation. Hypertens Res 2013; 36: 478-484.

3 Bramlage P, Pittrow D, Wittchen HU, Kirch W, Boehler S, Lehnert H, Hoefler M, Unger T, Sharma AM. Hypertension in overweight and obese primary care patients is highly prevalent and poorly controlled. Am J Hypertens 2004; 17: 904-910.
4 Must A, Spadano J, Coakley EH, Field AE, Colditz G, Dietz WH. The disease burden associated with overweight and obesity. JAMA 1999; 282: 1523-1529.

5 Wilson PW, D'Agostino RB, Sullivan L, Parise H, Kannel WB. Overweight and obesity as determinants of cardiovascular risk: the Framingham experience. Arch Intern Med 2002; 162: 1867-1872.

6 Oliva RV, Bakris GL. Blood pressure effects of sodium-glucose co-transport 2 (SGLT2) inhibitors. J Am Soc Hypertens 2014; 8: 330-339.

7 Vallon V, Platt KA, Cunard R, Schroth J, Whaley J, Thomson SC, Koepsell H, Rieg T. SGLT2 mediates glucose reabsorption in the early proximal tubule. J Am Soc Nephrol 2011; 22: 104-112.

8 Liakos A, Karagiannis T, Athanasiadou E, Sarigianni M, Mainou M, Papatheodorou K, Bekiari E, Tsapas A. Efficacy and safety of empagliflozin for type 2 diabetes: a systematic review and meta-analysis. Diabetes Obes Metab 2014; 16: 984-993.

9 Bode B, Stenlof K, Harris S, Sullivan D, Fung A, Usiskin K, Meininger G. Long-term efficacy and safety of canagliflozin over 104 weeks in patients aged 55-80 years with type 2 diabetes. Diabetes Obes Metab 2015; 17: 294-303.

10 Maliha G, Townsend RR. SGLT2 inhibitors: their potential reduction in blood pressure. J Am Soc Hypertens 2015; 9: 48-53.

11 Vasilakou D, Karagiannis T, Athanasiadou E, Mainou M, Liakos A, Bekiari E, Sarigianni M, Matthews DR, Tsapas A. Sodium-glucose cotransporter 2 inhibitors for type 2 diabetes: a systematic review and meta-analysis. Ann Intern Med 2013; 159: 262-274.

12 Bolinder J, Ljunggren O, Kullberg J, Johansson L, Wilding J, Langkilde AM, Sugg J, Parikh S. Effects of dapagliflozin on body weight, total fat mass, and regional adipose tissue distribution in patients with type 2 diabetes mellitus with inadequate glycemic control on metformin. J Clin Endocrinol Metab 2012; 97: 1020-1031.

13 Lambers Heerspink HJ, de Zeeuw D, Wie L, Leslie B, List J. Dapagliflozin a glucose-regulating drug with diuretic properties in subjects with type 2 diabetes. Diabetes Obes Metab 2013; 15: 853-862.

14 Lin B, Koibuchi N, Hasegawa Y, Sueta D, Toyama K, Uekawa K, Ma M, Nakagawa T, Kusaka H, Kim-Mitsuyama S. Glycemic control with empagliflozin, a novel selective SGLT2 inhibitor, ameliorates cardiovascular injury and cognitive dysfunction in obese and type 2 diabetic mice. Cardiovasc Diabetol 2014; 13: 148

15 Thomson SC, Rieg T, Miracle C, Mansoury H, Whaley J, Vallon V, Singh P. Acute and chronic effects of SGLT2 blockade on glomerular and tubular function in the early diabetic rat. Am J Physiol Regul Integr Comp Physiol 2012; 302: R75-R83.

16 Navar LG. The kidney in blood pressure regulation and development of hypertension. Med Clin North Am 1997; 81: 1165-1198.

17 Sufiun A, Rafiq K, Fujisawa Y, Rahman A, Mori H, Nakano D, Kobori H, Ohmori K, Masaki T, Kohno M, Nishiyama A. Effect of dipeptidyl peptidase-4 inhibition on circadian blood pressure during the development of salt-dependent hypertension in rats. Hypertens Res 2015; 38: 237-243.

18 Yagil Y, Mekler J, Wald H, Popovtzer MM, Ben-Ishay D. Sodium handling by the Sabra hypertension prone (SBH) and resistant (SBN) rats. Pflugers Arch 1986; 407: 547-551.

19 Chen J, Gu D, Huang J, Rao DC, Jaquish CE, Hixson JE, Chen CS, Chen J, Lu F, Hu D, Rice T, Kelly TN, Hamm LL, Whelton PK, He J, GenSalt Collaborative Research Group. Metabolic syndrome and salt sensitivity of blood pressure in non-diabetic people in China: a dietary intervention study. Lancet 2009; 373: 829-835.

20 Uzu T, Kimura G, Yamauchi A, Kanasaki M, Isshiki K, Araki S, Sugiomoto T, Nishio Y, Maegawa H, Koya D, Haneda M, Kashiwagi A. Enhanced sodium sensitivity and disturbed circadian rhythm of blood pressure in essential hypertension. $J$ Hypertens 2006; 24: 1627-1632.

21 Grempler R, Thomas L, Eckhardt M, Himmelsbach F, Sauer A, Sharp DE, Bakker RA, Mark M, Klein T, Eickelmann P. Empagliflozin, a novel selective sodium glucose cotransporter-2 (SGLT-2) inhibitor: characterisation and comparison with other SGLT-2 inhibitors. Diabetes Obes Metab 2012; 14: 83-90.

22 Rodriguez R, Viscarra JA, Minas JN, Nakano D, Nishiyama A, Ortiz RM. Angiotensin receptor blockade increases pancreatic insulin secretion and decreases glucose intolerance during glucose supplementation in a model of metabolic syndrome. Endocrinology 2012; 153: 1684-1695.

23 Hansen HH, Jelsing J, Hansen CF, Hansen G, Vrang N, Mark M, Klein T, Mayoux E. The sodium glucose cotransporter type 2 inhibitor empagliflozin preserves beta-cell mass and restores glucose homeostasis in the male zucker diabetic fatty rat. J Pharmacol Exp Ther 2014; 350: 657-664.

24 Oelze M, Kroller-Schon S, Welschof $P$, Jansen $T$, Hausding M, Mikhed $Y$, Stamm $P$ Mader M, Zinssius E, Agdauletova S, Gottschlich A, Steven S, Schulz E, Bottari SP, Mayoux E, Munzel T, Daiber A. The sodium-glucose co-transporter 2 inhibitor empagliflozin improves diabetes-induced vascular dysfunction in the streptozotocin diabetes rat model by interfering with oxidative stress and glucotoxicity. PLOS ONE 2014; 9: e112394.

25 Rafiq K, Nishiyama A, Konishi Y, Morikawa T, Kitabayashi C, Kohno M, Masaki T, Mori $\mathrm{H}$, Kobori H, Imanishi M. Regression of glomerular and tubulointerstitial injuries by dietary salt reduction with combination therapy of angiotensin II receptor blocker and calcium channel blocker in Dahl salt-sensitive rats. PLOS ONE 2014; 9: e107853.

26 Katayama T, Sueta D, Kataoka K, Hasegawa Y, Koibuchi N, Toyama K, Uekawa K, Mingjie M, Nakagawa T, Maeda M, Ogawa H, Kim-Mitsuyama S. Long-term renal denervation normalizes disrupted blood pressure circadian rhythm and ameliorates cardiovascular injury in a rat model of metabolic syndrome. J Am Heart Assoc 2013; 2: e000197.

27 Sueta D, Kataoka K, Koibuchi N, Toyama K, Uekawa K, Katayama T, Mingjie M, Nakagawa T, Waki H, Maeda M, Yasuda O, Matsui K, Ogawa H, Kim-Mitsuyama S. Novel mechanism for disrupted circadian blood pressure rhythm in a rat model of 
metabolic syndrome-the critical role of angiotensin II. J Am Heart Assoc 2013; 2: e000035

28 Pimenta E, Gaddam KK, Oparil S, Aban I, Husain S, Dell'Italia LJ, Calhoun DA. Effects of dietary sodium reduction on blood pressure in subjects with resistant hypertension: results from a randomized trial. Hypertension 2009; 54: 475-481.

29 Uzu T, Ishikawa K, Fujii T, Nakamura S, Inenaga T, Kimura G. Sodium restriction shifts circadian rhythm of blood pressure from nondipper to dipper in essential hypertension. Circulation 1997; 96: 1859-1862.

30 Zinman B, Wanner C, Lachin JM, Fitchett D, Bluhmki E, Hantel S, Mattheus M, Devins T, Johansen OE, Woerle HJ, Broedl UC, Inzucchi SEEMPA-REG OUTCOME Investigators. Empagliflozin, cardiovascular outcomes, and mortality in type 2 diabetes. N Engl J Med 2015; 373: 2117-2128.

31 Devenny JJ, Godonis HE, Harvey SJ, Rooney S, Cullen MJ, Pelleymounter MA. Weight loss induced by chronic dapagliflozin treatment is attenuated by compensatory hyperphagia in diet-induced obese (DIO) rats. Obesity 2012; 20: 1645-1652.

32 Suzuki M, Takeda M, Kito A, Fukazawa M, Yata T, Yamamoto M, Nagata T, Fukuzawa T, Yamane M, Honda K, Suzuki Y, Kawabe Y. Tofogliflozin, a sodium/glucose cotransporter
2 inhibitor, attenuates body weight gain and fat accumulation in diabetic and obese animal models. Nutr Diabetes 2014; 4: e125.

33 Rahmoune $\mathrm{H}$, Thompson PW, Ward JM, Smith CD, Hong G, Brown J. Glucose transporters in human renal proximal tubular cells isolated from the urine of patients with non-insulin-dependent diabetes. Diabetes 2005; 54: 3427-3434.

34 Kobori H, Nangaku M, Navar LG, Nishiyama A. The intrarenal renin-angiotensin system: from physiology to the pathobiology of hypertension and kidney disease. Pharmacol Rev 2007; 59: 251-287.

35 Koriyama $H$, Nakagami $H$, Nakagami F, Osako MK, Kyutoku M, Shimamura M, Kurinami H, Katsuya T, Rakugi H, Morishita R. Long-Term Reduction of high blood pressure by angiotensin II DNA vaccine in spontaneously hypertensive rats. Hypertension 2015; 66: 167-174.

36 Cherney DZ, Perkins BA, Soleymanlou N, Xiao F, Zimpelmann J, Woerle HJ, Johansen OE, Broedl UC, von Eynatten M, Burns KD. Sodium glucose cotransport-2 inhibition and intrarenal RAS activity in people with type 1 diabetes. Kidney Int 2014; 86: 1057-1058. 